\author{
LA GRANJA: \\ REVISTA DE \\ CIENCIAS DE LA VIDA \\ pISSN:1390-3799; eISSN:1390-8596 \\ http://doi.org/10.17163/lgr.n32.2020.02
}

\title{
RENDIMIENTO DEL MOTOR Y ANÁLISIS DE EMISIONES UTILIZANDO BIODIÉSEL DE NEEM Y JATROPHA
}

\author{
ENGINE PERFORMANCE AND EMISSION ANALYSIS USING NEEM AND \\ JATROPHA BLENDED BIODIÉSEL
}

\author{
Mehmood Ali ${ }^{1 *(0)}$ y Saqib Jamshed Rind ${ }^{2}$ (B)
}

\author{
${ }^{1}$ Departamento de Ingenieria Ambiental, NED University of Engineering and Technology, Karachi, Karachi City, Sindh 75270, \\ Pakistan. \\ 2 Departamento de Ingeniería Marina y Automotriz, NED University of Engineering and Technology, Karachi, Karachi City, \\ Sindh 75270, Pakistan. \\ *Autor para correspondencia: mehmood@neduet.edu.pk
}

Manuscrito recibido el 8 de mayo de 2020. Aceptado, tras revisión, el 6 de junio de 2020. Publicado el 1 de septiembre de 2020.

\begin{abstract}
Resumen
Este documento analiza la producción de biodiésel a partir de especies autóctonas de aceites de Jatropha curcas y Neem (Azadirachta indica), junto con el rendimiento del motor y las características de emisión de mezclas B10 a 1000 rpm. Los rendimientos de la producción de biodiésel fueron $90 \%$ y $68 \%$ en peso de Jatropha curcas y Neem (Azadirachta indica), respectivamente. Las tres mezclas preparadas de biodiésel fueron 10\% Biodiésel de Neem (NB10), 10\% de biodiésel de Jatropha (JB10) y 5\% de Jatropha + 5\% de Biodiésel de Neem (NJB10). La prueba de emisiones del motor mostró menos producción de monóxido de carbono con NB10 (94 $\pm 2,15$ ppm), seguida de JB10 $(100 \pm 2,44$ ppm) y NJB10 (121 \pm 3,65 ppm) en comparación con el diésel (135 \pm 2,18 ppm). Sin embargo, las emisiones de dióxido de carbono fueron más altas debido a las mejores características de combustión de las mezclas de biodiésel como NB10 (3,21\%), JB10 (3,06\%) y NJB10 (2,53\%) comparado con el diésel (2,13\%) por volumen. Las cantidades más bajas de emisiones de dióxido de azufre $\left(\mathrm{SO}_{2}\right)$ se observaron con el combustible de biodiésel mezclado, en comparación con el diésel mineral. Las emisiones de dióxido de nitrógeno $\left(\mathrm{NO}_{2}\right)$ fueron de $5 \mathrm{ppm}$ de diésel a $73^{\circ} \mathrm{C}$ de temperatura de escape, mientras que se incrementó a 8 ppm mediante el uso de biodiésel mezclado con NB10 debido a las altas temperaturas de escape de $85,33^{\circ} \mathrm{C}$. La potencia y la carga del motor producidos a partir de las muestras de biodiésel mezclado fueron ligeramente inferiores al diésel convencional en un $12 \%$ y un 7,7\%, respectivamente. Los resultados experimentales mostraron que el rendimiento del motor y la emisión del biodiésel de Neem (NB10) era mejor en comparación con otras mezclas de biodiésel.
\end{abstract}

Palabras clave: Biodiésel, Jatropha curcas, Neem (Azadirachta indica), emisiones del motor, rendimiento del motor. 


\begin{abstract}
This paper presents the production of biodiesel from indigenous species of Jatropha curcas and Neem (Azadirachta indica) oils, then its engine performance and emission characteristics of B10 blends measured at $1000 \mathrm{rpm}$. Biodiesel production yields were found $90 \%$ and $68 \%$ by weight from Jatropha curcas and Neem (Azadirachta indica), respectively. Three prepared biodiesel blends were 10\% Neem biodiesel (NB10), 10\% Jatropha biodiesel (JB10) and 5\% Jatropha $+5 \%$ Neem biodiesels (NJB10). The engine emission test showed less carbon monoxide production from NB10 $(94 \pm 2.15 \mathrm{ppm})$, followed by JB10 $(100 \pm 2.44 \mathrm{ppm})$ and NJB10 $(121 \pm 3.65 \mathrm{ppm})$ as compared to diesel (135 $\pm 2.18 \mathrm{ppm}$ ). However, the carbon dioxide emissions were found higher due to the better combustion characteristics of biodiesel blends as NB10 (3.21\%), JB10 (3.06\%) and NJB10 $(2.53 \%)$ than diesel $(2.13 \%)$ by volume. The reduced amounts of sulphur dioxide $\left(\mathrm{SO}_{2}\right)$ emissions were found with blended biodiesel fuel in comparison to mineral diesel. Nitrogen dioxide $\left(\mathrm{NO}_{2}\right)$ emissions were $5 \mathrm{ppm}$ from diesel at $73^{\circ} \mathrm{C}$ exhaust temperature, while it was increased by using blended biodiesel, to $8 \mathrm{ppm}$ with NB10 due to higher exhaust temperatures $85,33^{\circ} \mathrm{C}$. The measured engine power and torque produced from the blended biodiesel samples were slightly lower than the conventional diesel by $12 \%$ and $7.7 \%$, respectively. The experimental results showed that an engine performance and emission characteristic of Neem biodiesel (NB10) was better as compared to other biodiesel blends.
\end{abstract}

Keywords: Biodiesel, Jatropha curcas, Neem (Azadirachta indica), engine emissions, engine performance.

Forma sugerida de citar: Ali, M. y Jamshed-Rind, S. (2020). Rendimiento del motor y análisis de emisiones utilizando biodiésel de Neem y Jatropha. La Granja: Revista de Ciencias de la Vida. Vol. 32(2):19-29. http://doi.org/10.17163/lgr.n32.2020.02.

IDs Orcid:

Mehmood Ali: http:/ /orcid.org/0000-0002-1804-2677

Saqib Jamshed Rind: http:/ / orcid.org/0000-0003-4881-0419 


\section{Introducción}

El cambio climático y los problemas del calentamiento global son causados por las emisiones de gases de efecto invernadero (GEI) como el dióxido de carbono, el metano y el óxido nitroso en la atmósfera debido a las actividades inducidas por el ser humano por la quema de combustibles fósiles utilizados en el sector del transporte y la generación de energía (Climate Change Indicators, 2015). Los dos biocombustibles líquidos más comunes para el transporte (el bioetanol y el biodiésel) pueden sustituir a la gasolina derivada del petróleo y a los combustibles diésel, que producen emisiones de gases nocivos con impactos perjudiciales en el medio ambiente. El bioetanol y el biodiésel son combustibles alternativos producidos casi en su totalidad a partir de cultivos de semillas de aceite vegetal que tienen beneficios ambientales. La mayor diferencia entre los biocombustibles y las materias primas del petróleo es la concentración de contenido de oxígeno, que hace que los biocombustibles sean respetuosos con el medio ambiente, con una combustión completa que produce dióxido de carbono en lugar de monóxido de carbono, causando un impacto negativo en la salud de los seres humanos (Chauhan y Shukla, 2011).

El biodiésel, como combustible biodegradable y alternativo, tiene mayor importancia debido al agotamiento de las reservas de petróleo crudo y sus beneficios con el medio ambiente (Berchmans e Hirata, 2008). De hecho, el combustible biodiésel es una forma de combustible renovable que se puede obtener de varias reservas, incluidos los cultivos de semillas de aceites comestibles y no comestibles, aunque también se puede producir a partir de aceites no comestibles, por ejemplo, mahua, neem, karanja y Jatropha curcas para evitar problemas con los cultivos alimentarios comestibles (Hassan y Kalam, 2013). El combustible biodiésel en el motor diésel no requiere modificaciones del motor, provee una reducción significativa de las emisiones de óxidos de azufre $\left(S O_{x}\right)$, monóxido de carbono $(C O)$ y partículas (PM) con otros beneficios como mayor punto de ignición y menor contenido aromático en los gases de escape, debido a la combustión completa del combustible (Ali y Shaikh, 2012).

En investigaciones previas, el éster metílico de Pongamia, Jatropha curcas y Neem se produjo por reacción de transesterificación en un estudio experimental llevado a cabo para probar las características de emisión de diferentes mezclas $(B 10, B 20$, y B40) en comparación con el diésel de petróleo. Las muestras de combustible mezclado mostraron menores emisiones de humo, óxido de carbono, monocarburos e hidrocarburos no quemados (HC) en comparación con el diésel de petróleo (Rao, Rao y Reddy, 2008). Otra investigación mostró que las misiones de escape del biodiésel de neem B30 tenían menor opacidad de humo (5\%) que el combustible diésel regular $(55 \%)$ a potencia máxima de freno $(5 \mathrm{~kW})$. Del mismo modo, el monóxido de carbono $(\mathrm{CO})$ es uno de los productos intermedios formados durante la reacción de la combustión de hidrocarburos en el cilindro del motor, y se observó que la cantidad de $C O$ producido disminuye con el aumento de la carga en el motor para el diésel y varias mezclas de biodiésel de neem. Se observó que las emisiones de hidrocarburos no quemados (HC) aumentan en comparación con el incremento de la carga del motor para el diésel y las mezclas de biodiésel de neem, debido a un mayor consumo de combustible a altas cargas del motor, con una combustión incompleta. Sin embargo, se encontró que las emisiones de HC disminuyen con el aumento en el porcentaje de biodiésel en las mezclas, debido a la disponibilidad de más porcentaje de oxígeno que conduce a la combustión completa de combustibles (Mall, 2015).

La mayor parte de la producción de energía en Pakistán se satisface mediante la quema de combustibles derivados del petróleo crudo, que son recursos no renovables. Actualmente el país se enfrenta a un déficit de electricidad entre 6.000 y 7.000MW (Yuosafzai, 2018). La factura de importación de petróleo en Pakistán aumentó de 7.400 millones de dólares americanos en 2015-16 a 9.100 millones de dólares americanos en el año fiscal 2016-17, como resultado del aumento de los precios internacionales del crudo y el incremento de las importaciones de productos petrolíferos. Por lo tanto, se supone que la demanda de petróleo crudo tendrá una tendencia creciente en el futuro para la generación de energía y el sector del transporte en el país (Pervaz, 2018). Sin embargo, para el futuro sostenible a través de las energías renovables es necesario reducir la dependencia del petróleo crudo importado, estas medidas no sólo ayudarían a proteger valiosos intercambios extranjeros del país, sino que también ayudarían a mitigar el cambio climático y 
los problemas del calentamiento global (Ali, 2016). Además, la Junta de Desarrollo de Energía Alternativa de Pakistán (AEDB) tomó una iniciativa en el marco de su Programa Nacional de Biodiésel de minimizar la cantidad de consumo de diésel $(10 \%$ del volumen) del consumo total de diésel para el año 2025 por medio de un combustible biodiésel alternativo (Ahmed y col., 2015).

El biodiésel es un combustible carbono neutro que no causa daños al medio ambiente, pues tiene pocas emisiones de gases tóxicos. La investigación llevada a cabo anteriormente tuvo un alcance limitado sobre los combustibles de biodiésel mezclados de Neem y Jatropha curcas en relación con el rendimiento del motor y las características de emisión. Por lo tanto, el objetivo de este estudio fue caracterizar el biodiésel producido a partir de aceites de Neem y Jatropha, seguido por el rendimiento del motor y las características de emisión de Neem y Jatropha curcas mezcladas con muestras de combustible biodiésel con el fin de cumplir con el objetivo AEDB de utilizar un 10\% de biodiésel mezclado para el año 2025 en Pakistán.

\section{Materiales y métodos}

El aceite de semilla vegetal no comestible de Jatropha curcas se obtuvo del Centro de Investigación de Zonas Áridas del Consejo de Investigación Agrícola de Pakistán, Umerkot, mientras que el aceite de neem (Azadirachta indica) se extrajo con un expulsor mecánico de semillas de aceite disponibles localmente. Todos los experimentos se llevaron a cabo en el Departamento de Ingeniería Ambiental y el Departamento de Automoción e Ingeniería Marina, NED University of Engineering Technology, Karachi, Pakistán. La temperatura y la humedad en el laboratorio se midieron como $28^{\circ} \mathrm{C}$ y $45 \%$ de humedad con (Termómetro Digital del Medidor de Humedad de Temperatura, China).

\subsection{Prueba de ácidos}

Para ambos aceites no comestibles, el contenido de ácidos grasos libres (FFA) \% y la prueba de valor ácido se midieron siguiendo el método de valoración estándar (Método Oficial de la Sociedad Americana de Químicos del Aceite Ca 5a-40) mencionado en la literatura (Berchmans e Hirata, 2008). Según la documentación, si los aceites vegetales tienen un contenido de FFA superior al $1 \%$ en peso, producirá jabón y un bajo rendimiento del producto biodiésel. Por lo tanto, generalmente se adopta el proceso de dos pasos (es decir, esterificación ácida y transesterificación catalizada de base) para reducir el aceite FFA y producir biodiésel (Berchmans e Hirata, 2008).

\subsection{Dos pasos (esterificación ácida y transesterificación catalizada de base)}

El proceso de dos pasos, proceso de esterificación catalizada por ácido y seguido por el proceso de transesterificación catalizada de base se muestra en la literatura (Berchmans e Hirata, 2008). El primer paso se llevó a cabo con $0,60 \mathrm{p} / \mathrm{p}$ de metanol a las relaciones de aceite en presencia de $1 \% \mathrm{p} / \mathrm{p} \mathrm{H}_{2} \mathrm{SO}_{4}$ (BDH, Inglaterra) como catalizador ácido en reacción de 1 hora a $50^{\circ} \mathrm{C}$ utilizando un calentador de placa caliente con agitador magnético que funciona a $400 \mathrm{rpm}$ (Wise stir, MSH-20A, Daihan Scientific, Corea). Después de la reacción, la mezcla se detuvo durante 2 horas y la mezcla de agua de metanol recogida en la capa superior se eliminó con la ayuda de una pipeta. El segundo paso fue la transesterificación catalizada base utilizando metanol de 0,24 $\mathrm{w} / \mathrm{w}(\mathrm{BDH}$, Inglaterra) al aceite y 1,4\% w/w $\mathrm{NaOH}$ (Merck, Alemania) al aceite como catalizador alcalino para producir biodiésel a $65^{\circ} \mathrm{C}$ durante 2 horas de tiempo de reacción, utilizando un calentador magnético de placa caliente con agitador con una velocidad de mezcla de $400 \mathrm{rpm}$ (Berchmans e Hirata, 2008). Las dos capas se formaron después de la reacción, es decir, el biodiésel (capa superior) y la glicerina (capa inferior) se separaron usando un embudo separador (1L). La fase de biodiésel y la fase de glicerina se lavaron con un $10 \%$ de volumen en agua destilada caliente a $70^{\circ} \mathrm{C}$ y luego se secaron en un horno convencional (YCO-N01, Gemany Industrial Corp., Taiwán) durante 1 hora a $80^{\circ} \mathrm{C}$ (Ali, Naqvi y Watson, 2018). La cantidad de biodiésel y glicerina producida después de la separación y el secado se recogieron en un vaso de precipitados (50 $\mathrm{ml}$ ) y la cantidad se midió en gramos con la ayuda de un peso (AB 304-S, Mettler Tolendo, Suiza). 


\subsection{Propiedades fisicoquímicas del biodié- sel producido}

Las siguientes propiedades fisicoquímicas del biodiésel producido y los resultados se presentan como media \pm desviación estándar, para el tamaño de la muestra $(n=3)$.

\subsubsection{Densidad:}

La densidad del aceite extraído y el biodiésel producido se midieron en $\mathrm{g} / \mathrm{mL}$ con un densímetro (DA130 N, Kyoto Electronics Manufacturing Co. Ltd, Japan).

\subsubsection{Viscosidad cinemática:}

La viscosidad cinemática en $\mathrm{mm}^{2} / \mathrm{seg}$ de los aceites vegetales (aceites Jatropha curcas y Neem) y sus muestras de biodiésel se midieron utilizando un viscosímetro cinemático/dinámico (VDM-300, AS Lemis, UE).

\subsubsection{Valor calorífico:}

El valor calorífico en $\mathrm{MJ} / \mathrm{kg}$ de los aceites de Jatropha curcas y Neem y sus muestras de biodiésel mezclado se determinaron con un calorímetro de bomba de oxígeno (IKA C200, Alemania) según el método estándar ASTM D2015.

\subsubsection{Punto de ignición:}

El punto de igición del biodiésel de Jatropha curcas y Neem y las muestras de combustible mezclado se midieron con un probador de puntos de inflamación (AD 0093-710, SCAVINI, Italia) según el método estándar ASTM D93.

\subsection{El contenido de ésteres metílicos (ácido oleico)}

La composición de ácido oleico en las muestras de aceite de Jatropha y Neem se determinaron utilizando GC-FID (GC-2014, Shimadzu, Japón) equipado con un detector de ionización de llama en el Instituto de Ingeniería Química y Tecnología, Universidad del Punjab, Lahore.

\subsection{Biodiésel -relaciones de mezcla de dié- sel}

Las muestras de combustible $(1 \mathrm{~L}=1000 \mathrm{ml})$ se prepararon mezclando el biodiésel producido con el diésel convencional adquirido de la estación de combustible operada por la empresa de Pakistan State Oil (PSO) en las siguientes proporciones, como se muestra en la Tabla 1 y según el requisito de AEDB para mezclar el diésel convencional con el 10\% de combustible de biodiésel.

\subsection{Protocolo de prueba de emisiones y rendimiento del motor}

Las características de emisión de las muestras de combustible preparadas se midieron con el funcionamiento de un motor diésel con las especificaciones mencionadas en la Tabla 2, a una velocidad constante de 1000 rpm en el Departamento de Automoción e Ingeniería Marina de la Universidad NED. La medición de la concentración de emisiones de gases de dióxido de carbono $\left(\mathrm{CO}_{2}\right)$, monóxido de carbono $(\mathrm{CO})$, óxidos de nitrógeno $\left(\mathrm{NO}_{\mathrm{X}}\right)$, dióxido de azufre $\left(\mathrm{SO}_{2}\right)$ y contenido de oxígeno $\left(\mathrm{O}_{2}\right)$ se hizo utilizando el analizador de gases de combustión (340, Testo Instruments Ltd, Alemania). Los resultados de la emisión de gases se presentan como (media \pm desviación estándar) para el tamaño de la muestra $n=3$.

El rendimiento del motor se midió para el combustible $100 \%$ diésel, biodiésel NB10 neem, biodiésel JB10 Jatropha y biodiésel NJB10 (mezcla de neem y Jatropha). El rendimiento del motor se estudió a una velocidad constante de $1000 \mathrm{rpm}$ siguiendo los procedimientos mencionados en la literatura (Calder, Roy y Wang, 2018). Después de que el motor alcanzara su condición de trabajo estabilizada, el par de carga de retención externo (10\%) se equipó con un dinamómetro de freno de agua conectado con un controlador instalado en la liberación de carga de agua para controlar la carga del motor. La potencia de freno del motor se calculó utilizando la Ecuación 1 , donde el par de carga se mide en $N-m$ y la velocidad del motor en revolución por minuto.

Potencia de freno $(h p)=\frac{\text { Carga } \times \text { Velocidad del motor }}{5252}$ 
Tabla 1. Relación de las muestras de combustible preparadas.

\begin{tabular}{cccc}
\hline Muestra & $\begin{array}{c}\text { Diésel } \\
\text { mineral }(\mathbf{m L})\end{array}$ & $\begin{array}{c}\text { Biodiésel } \\
\text { de Neem }(\mathbf{m L})\end{array}$ & $\begin{array}{c}\text { Biodiésel de } \\
\text { Jatropha }(\mathbf{m L})\end{array}$ \\
\hline Diésel fuel & 1000 & - & - \\
NB10 & 900 & 100 & - \\
JB10 & 900 & - & 100 \\
NJB10 & 900 & 50 & 50 \\
\hline
\end{tabular}

Tabla 2. Especificaciones del motor utilizadas en este estudio.

\begin{tabular}{cc}
\hline Motor & Rotronics, France \\
\hline No. de cilindros & 2 \\
Volumen de los cilindros & $380 \mathrm{cc}$ \\
Velocidad máxima & $2700 \mathrm{rpm}$ \\
Potencia de salida máxima & $2.0 \mathrm{~kW}$ \\
Calibre & $62 \mathrm{~mm}$ \\
Tiempos & $72 \mathrm{~mm}$ \\
Tipo de motor & 4 tiempos, inyección directa, aire frío \\
Radio de compresión & $8.5: 1$ \\
\hline
\end{tabular}

2.7 Estimación del potencial de la producción de biodiésel para satisfacer los requisitos locales

La producción de biodiésel de cultivos de aceite de neem y aceite de cocina residual se calculó sobre la base de la tierra disponible en el país para satisfacer la demanda local anual.

\section{Resultados y discusión}

\subsection{Rendimiento del biodiésel y glicerol}

La cantidad medida de biodiésel y glicerol producido a partir del aceite de Jatropha curcas fue $90 \%$ $(36,28 \mathrm{~g} / 40 \mathrm{~g}$ de aceite) y $11 \%(4,36 \mathrm{~g} / 40$ aceite), respectivamente. Mientras que la cantidad de biodiésel y glicerol producido a partir del aceite de Neem fue $68 \%(27,49 \mathrm{~g} / 40 \mathrm{~g}$ de aceite $)$ y $30 \%(12,50$ $\mathrm{g} / 40$ aceite), respectivamente.

\subsection{Propiedades físicas y químicas del com- bustible}

Las propiedades del biodiésel de Jatropha curcas y Neem se determinaron siguiendo las normas y procedimientos ASTM que se presentan en la Tabla 3 y luego se compararon con la norma internacional de biodiésel (ASTM D 6751). Se observó que la viscosidad cinemática del biodiésel de Neem estuvo más baja, es decir, 2,91 $\mathrm{mm}^{2} / \mathrm{seg}$ en comparación con el biodiésel de Jatropha curcas 4,18 $\mathrm{mm}^{2} / \mathrm{seg}$. La viscosidad cinemática reducida es buena en términos de calidad del combustible biodiésel, pero el rendimiento del biodiésel de Neem fue un 24,33\%, menor en comparación con el rendimiento del biodiésel de Jatropha.

\subsection{Resultados del contenido de oleato de metilo}

La concentración de oleato de metilo en \% en peso se midió con el análisis GC-MS, y los resultados mostraron la concentración de oleato de metilo en el aceite de Jatropha curcas $(41,12 \% \mathrm{wt})$ y el aceite de Neem $(50,77 \% w t)$, respectivamente. Los resultados mostraron que el aceite de Neem tiene un valor comparable al aceite de Jatropha, con una propiedad más favorable con un tiempo de almacenamiento de biodiésel más largo, con una buena estabilidad a la oxidación y una disminución del punto de tapón del filtro frío durante los inviernos, manteniendo el biodiésel en fase líquida para una adecuada atomización. De igual forma, se recomienda en el futuro medir el perfil completo de ácidos grasos libres de ambas muestras de aceite vegetal. 
Tabla 3. Propiedades fisicoquímicas de los aceites de semillas vegetales y su biodiésel producido.

\begin{tabular}{lcccc}
\hline Parámetros & $\begin{array}{c}\text { Jatropha } \\
\text { Curcas }\end{array}$ & $\begin{array}{c}\text { Neem } \\
\text { (Azadirachta indica) }\end{array}$ & Método de prueba & $\begin{array}{c}\text { ASTM D 6751 } \\
\text { Biodiésel standard }\end{array}$ \\
\hline Densidad del aceite $\left(\mathrm{g} / \mathrm{cm}^{3}\right)$ & 0,877 & 0,914 & ASTM D1298 & - \\
Viscosidad biológica del aceite & 44,5 & 33,37 & ASTM D 445 & - \\
$\left(\mathrm{mm}^{2} / \mathrm{sec}\right)$ a $40^{\circ} \mathrm{C}$ & 36,28 & 27,49 & - & - \\
Rendimiento del biodiésel $(\mathrm{g})$ & 0,884 & 0,875 & ASTM D1298 & $0,86-0,90^{*}$ \\
Densidad del biodiésel $\left(\mathrm{g} / \mathrm{cm}^{3}\right)$ & 4,18 & 2,91 & ASTM D445 & $1,9-6,0$ \\
Viscosidad cinemática del & 4,36 & 12,5 & - & - \\
biodiésel $\left(\mathrm{mm}^{2} / \mathrm{sec}\right)$ & 38,96 & 39,67 & ASTM D240 & - \\
Rendimiento de la glicerina $(\mathrm{g})$ & 130 & ASTM D93 & $130^{\circ} \mathrm{C}(\mathrm{m}$ mínimo) \\
\hline Valor calórico del biodiésel $(\mathrm{MJ} / \mathrm{kg})$ & & & \\
Punto de ingnición $\left({ }^{\circ} \mathrm{C}\right)$ del biodiésel & 120 & & & \\
\hline
\end{tabular}

*Según la norma europea de biodiésel EN 14214

\subsection{Resultados de las pruebas de emisiones del motor}

Se trazó el perfil de emisiones de gases de diferentes mezclas de combustible biodiésel para comparar la diferencia entre sus características de emisión. La Figura 1 muestra el porcentaje de contenido de oxígeno presente en las emisiones de gases de escape y se observó que el diésel mineral $(17,12 \pm 0,05 \%)$ presenta más contenido de oxígeno en comparación con las muestras de combustible mezclado con biodiésel. La posible razón podría ser debido a la alta temperatura y al alto contenido de oxígeno presente en el combustible biodiésel, y que el exceso de oxígeno presente en el biodiésel se utilizó en la producción de óxidos de nitrógeno $\left(N O_{x}\right)$. Generalmente el exceso de oxígeno no reacciona con nitrógeno en el cilindro del motor, pero reacciona con nitrógeno atmosférico a altas temperaturas de escape ((Nair, Kaviti y Daram, 2017).

Del mismo modo, el perfil de temperatura de escape del gas de combustión del motor diésel que funciona en diferentes mezclas de combustible (Figura 2) mostró que se obtuvieron temperaturas más altas a partir de la combustión del combustible de la mezcla de biodiésel en comparación con el combustible diésel. Esto se debe a la presencia de propiedades oxigenadas del biodiésel con la combustión completa, lo que resulta en temperaturas de escape más altas (es decir, $14 \%$ y $8,75 \%$ de combustibles de biodiésel mezclados de neem y jatropoha superiores al diésel mineral). Un estudio previo sobre biodiésel de palma mostró una temperatura de gases de escape 5,6\% mayor que el diésel de petróleo.
La mejor característica de combustión del biodiésel con mayor temperatura del cilindro se debe al efecto del exceso del contenido de oxígeno presente en su composición (Arunkumar y col., 2018).

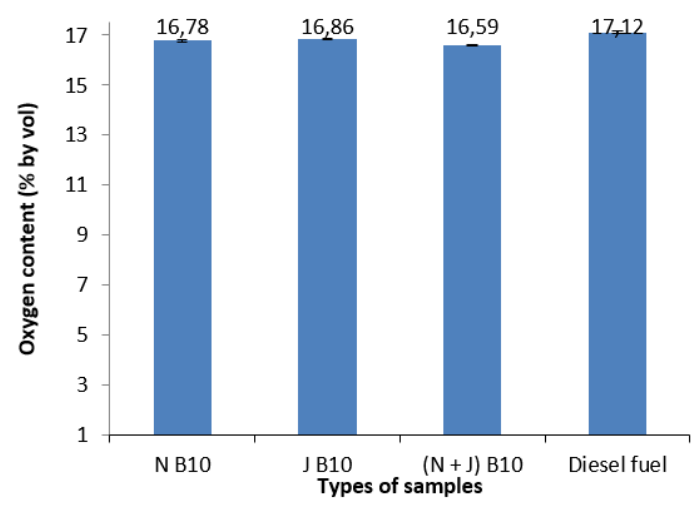

Figura 1. Contenido de oxígeno en las emisiones de gases de escape.

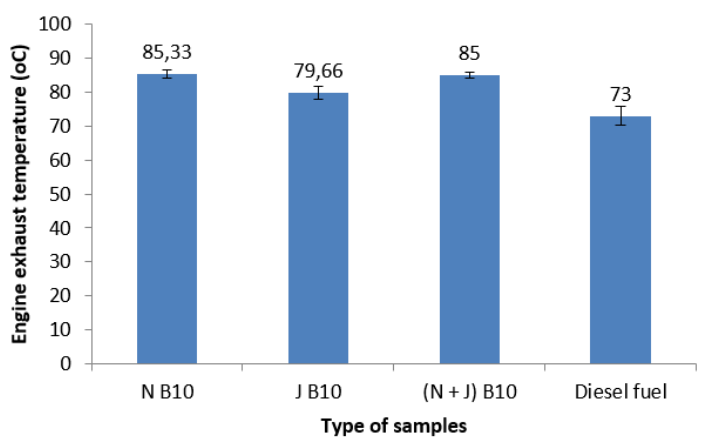

Figura 2. Temperaturas de escape con diferentes mezclas de combustible. 
La Figura 3 muestra la emisión de monóxido de carbono $(\mathrm{CO})$ de diferentes muestras de combustible, mostrando menos emisiones de $C O$ de combustibles mezclados con biodiésel (tales como NB10= $94 \pm 2,15, \mathrm{JB} 10=100 \pm 2,44$ and NJB10 $=121 \pm 3.65$ ppm) en comparación con el diésel mineral (135 \pm 2,18 ppm). De acuerdo a lo observado en la literatura, la razón principal de las mayores emisiones de $\mathrm{CO}$ de la combustión de diésel mineral se debe a su combustión incompleta (Nair, Kaviti y Daram, 2017). Por lo tanto, la presencia de un mayor porcentaje de oxígeno en el biodiésel conduce a un proceso de combustión más completo, lo que resulta en menores emisiones de $C O$ (Dincer, 2008).

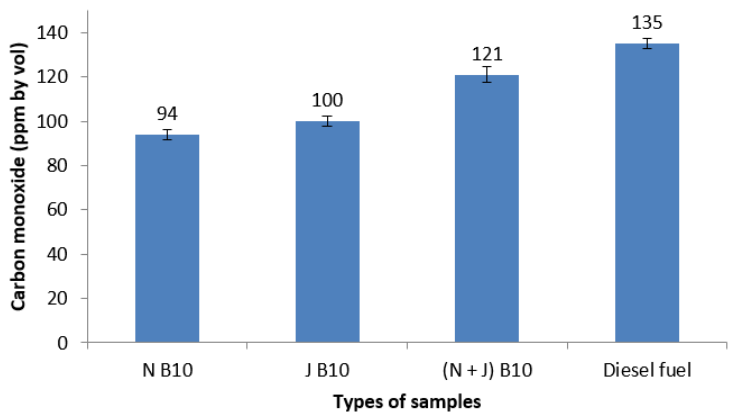

Figura 3. Contenido de monóxido de carbono en las emisiones de escape.

Los resultados actuales de las emisiones de dióxido de carbono $\left(\mathrm{CO}_{2}\right)$ se encontraron más altos en las muestras de combustible mezclado con biodiésel en comparación con el combustible diésel mineral (Figura 4). El biodiésel de Neem NB10 mostró mayores emisiones de $\mathrm{CO}_{2}$ en contraste con el biodiésel JB10 de Jatropha. Las emisiones de CO se redujeron para la mezcla de biodiésel de Neem (NB10) en un volumen del 30,37\% en comparación con el diésel mineral. El valor de emisión de $C O$ se encontró cerca con una reducción media para Neem B10 en un volumen del $26 \%$, como se menciona en la literatura (Nair, Kaviti y Daram, 2017).

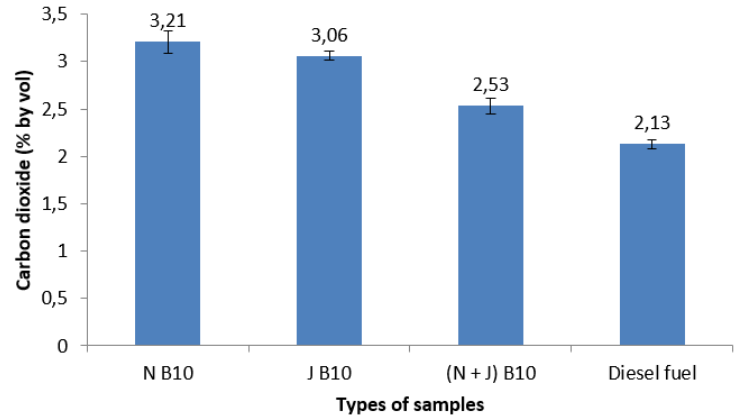

Figura 4. Contenido de dióxido de carbono en las emisiones de escape.

La Figura 5 muestra las emisiones de dióxido de nitrógeno $\left(N O_{x}\right)$ de la combustión de diferentes muestras de combustible mezclado. Se observó que las emisiones de $N O_{x}$ fueron mayores con respecto a las muestras de combustible mezclado con biodiésel, en comparación con el diésel mineral (es decir, $37,5 \%$ de NB10 y $28,57 \%$ de JB10, respectivamente). También en otras investigaciones se observó un aumento del $20 \%$ en las emisiones de NOx de neem B10 en comparación con el combustible diésel (Nair, Kaviti y Daram, 2017). La temperatura más alta es el resultado de la combustión de biodiésel, que produce $N O_{x}$ con una reacción entre el exceso de contenido de oxígeno presente en las emisiones de combustible mezclado con biodiésel y nitrógeno atmosférico. Las emisiones de dióxido de azufre $\left(S O_{x}\right)$ se presentan en la Figura 6, y los resultados mostraron una reducción de las emisiones de $S O_{x}$ en más del $38,46 \%$ de NB10 y del 34,61\% de JB10, respectivamente, en comparación con el diésel mineral. Resulta ventajoso utilizar combustible mezclado con biodiésel con bajo contenido de azufre para reducir la contaminación del aire que conlleva al calentamiento global y los problemas de lluvia ácida.

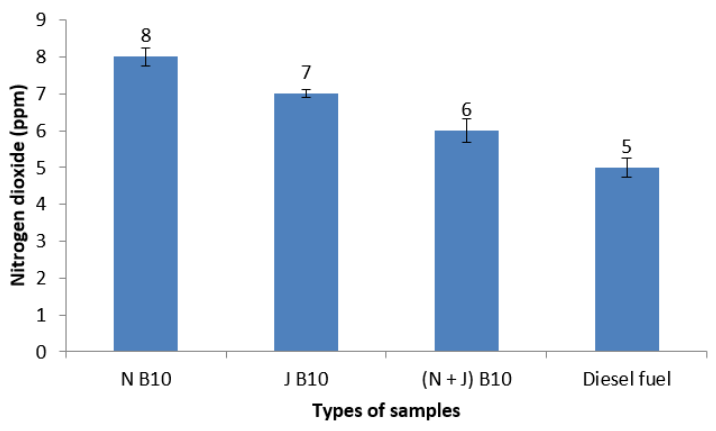

Figura 5. Contenido de dióxido de nitrógeno en las emisiones de escape. 


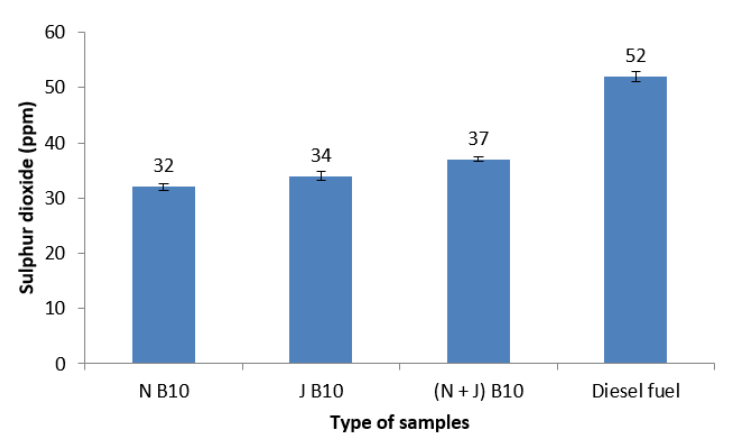

Figura 6. Contenido de dióxido de azufre en las emisiones de escape.

\subsection{Mediciones de potencia del motor y freno}

La variación de la potencia del freno en las diferentes muestras de combustible mezclado (diésel mineral, NB10, JB10 y NJB10) a una velocidad constante del motor de $1000 \mathrm{rpm}$ se presenta en la Tabla 4. Los resultados mostraron menor potencia generada a partir del combustible biodiésel mezclado en comparación con el diésel de petróleo debido a su menor valor calorífico ( 38 a $39 \mathrm{MJ} / \mathrm{kg}$ ) y en comparación con el diésel mineral $(42 \mathrm{MJ} / \mathrm{kg})$, información que también se menciona en la literatura (Chakrabarti y Ali, 2008). Además, se observó que la carga del motor y la potencia generadas por el biodiésel Neem NB10 fueron mayores en comparación con el biodiésel JB10 de Jatropha, debido al mayor valor calorífico del biodiésel de Neem $(39,67 \mathrm{MJ} / \mathrm{kg})$ en contraste con el biodiésel de Jatropha $(38,96 \mathrm{MJ} / \mathrm{kg})$. La combustión del diésel mineral produjo una carga más alta durante las pruebas del motor en comparación con las diferentes mezclas de biodiésel, debido a su mayor valor calorífico $(42 \mathrm{MJ} / \mathrm{kg}$ ) (Cha- krabarti y Ali, 2008). Los resultados indicaron que el B10 tiene un rendimiento más cercano del motor al diésel mineral debido a su viscosidad cinemática en comparación con el diésel mineral (Chakrabarti y Ali, 2009). La potencia generada por el biodiésel de Neem NB10, Jatropha JB10 y el biodiésel mixto Neem / Jatropha NJB 10 fueron de 7,7\%, un 16,4\% y un $12 \%$, respectivamente, menor que el diésel mineral.

\subsection{Estimación de la producción de biodié- sel para cumplir con los requisitos loca- les}

Las semillas de Neem (Azadirachta indica) contienen aproximadamente un $20-35 \%$ de contenido de aceite por peso y producen unos $2670 \mathrm{~kg}$ de aceite/hectárea (Aransiola y col., 2019). Según la literatura, Pakistán consta de tierras áridas/ semiáridas y marginales de aproximadamente 350.000 acres (141640 hectáreas) que pueden utilizarse para sembrar cultivos no comestibles para la producción de biodiésel (Ali, 2016). La Tabla 5, muestra que la producción de aceite de Neem en el país es de $378178,8 \mathrm{~kg}$ al año (es decir, 0,3781 millones de toneladas) obtenida, multiplicando los $2670 \mathrm{~kg}$ de petróleo/hectárea con la superficie marginal de tierra de 350.000 hectáreas. Se supone que el $90 \%$ en peso del aceite de neem producido al año se convierte en biodiésel, representando 0,3402 millones de toneladas, mientras que el $10 \%$ en peso se convierte en subproductos de glicerina (es decir, 0,0378 millones de toneladas). Además, según la literatura (Qamar y col., 2020) Pakistán tiene una capacidad de producir 468.842 toneladas $(0,4688$ millones de toneladas) de biodiésel anualmente utilizando aceite de cocina residual (ACR).

Tabla 4. Carga del motor y potencia de frenado correspondiente a una velocidad constante (1000 rpm).

\begin{tabular}{ccc}
\hline Muestras & $\begin{array}{c}\text { Carga del motor } \\
(\mathbf{N}-\mathbf{m})\end{array}$ & $\begin{array}{c}\text { Potencia de frenado } \\
(\mathbf{h p})\end{array}$ \\
\hline Combustible diesél & 2,854 & 0,543 \\
NB10 & 2,634 & 0,501 \\
JB10 & 2,387 & 0,454 \\
NJB10 & 2,514 & 0,478 \\
\hline
\end{tabular}


Tabla 5. Estimación de la producción local de biodiésel al año.

\begin{tabular}{llc}
\multicolumn{3}{c}{ Stadísticas de la producción local por año } \\
\hline (1) & $\begin{array}{l}\text { Demanda de petrodiésel al año } \\
\text { (millones de toneladas) }\end{array}$ & 6,764 \\
(2) & $\begin{array}{l}\text { Requisito de biodiésel mezclado (B10) } \\
\text { al año (millones de toneladas) }\end{array}$ & 0,6764 \\
(3) & $\begin{array}{l}\text { Producción de aceite de Neem al año } \\
\text { (millones de toneladas) }\end{array}$ & 0,3781 \\
(4) & $\begin{array}{l}\text { Producción estimada de biodiésel de neem } \\
\text { al año (millones de toneladas) }\end{array}$ & 0,3402 \\
(5) & $\begin{array}{l}\text { Producción estimada de biodiésel de la ACR } \\
\text { al año (millones de toneladas) }\end{array}$ & 0,4688 \\
(6) & $\begin{array}{l}\text { Cantidad excedente de producción de biodiésel (B10) } \\
\text { al año (millones de toneladas) }\end{array}$ & 0,1326 \\
\hline
\end{tabular}

La demanda total de diésel de alta velocidad en el país por año es de 6.674 millones de toneladas (DAWN, 2019) y si se utiliza biodiésel mezclado (B10) en el país, la cantidad requerida será de 0,6764 millones de toneladas al año para cumplir con el requisito AEDB de utilizar biodiésel mezclado B10. Para satisfacer la cantidad requerida de biodiésel, la diferencia entre la columna (2) menos las cantidades estimadas de biodiésel de Neem (columna 4) y el biodiésel de aceite de cocina residual (columna 5) da una cantidad mayor de producción de biodiésel (0,1326 millones de toneladas) en el país al año, sin competir con los cultivos comestibles de aceite.

\section{Conclusiones}

Los aceites de semillas vegetales de Jatropha y Neem se pueden convertir en biodiésel y biodiésel mezclado (B10), y pueden utilizarse como combustible alternativo en motores diésel para cumplir con el objetivo fijado por la Junta de Desarrollo de Energía Alternativa. La emisión de monóxido de carbono $(\mathrm{CO})$ de diferentes muestras de combustible mostró menos emisiones de $C O$ de combustibles mezclados con biodiésel.

La cantidad de concentración de $\mathrm{CO}_{2}$ mostró una tendencia creciente en las emisiones de escape con respecto al aumento de la cantidad de biodiésel en la mezcla de combustible, convirtiendo más $\mathrm{CO}$ en $\mathrm{CO}_{2}$. Las emisiones de $\mathrm{NO}_{x}$ fueron más altas con una relación creciente de combustible biodiésel con el diésel mineral. Los resultados indicaron que B10 tiene un rendimiento más cercano del motor con el diésel mineral, esto se debe principalmente a su viscosidad cinemática comparable. Se observó menos potencia del motor generada por el biodiésel Neem NB10, Jatropha JB10 y el biodiésel mixto Neem/Jatropha NJB10, es decir, el 7,7\%, el 16,4\% y el $12 \%$, respectivamente, en comparación con el diésel mineral.

La producción estimada de biodiésel en el país a partir de los cultivos de aceite de Neem y el aceite de cocina residual se encuentra produciendo una cantidad excedente de biodiésel (0,1326 millones de toneladas al año) para satisfacer la demanda local de utilizar combustible B10 en el futuro.

\section{Agradecimientos}

Los autores agradecen a la Universidad NED de Ingeniería y Tecnología, Karachi, Pakistán por proporcionar las instalaciones del laboratorio. Además, hacen un agradecimiento especial al Instituto de Investigación de la Zona árida (Consejo de Investigación Agrícola de Pakistán, Umerkot, Pakistán) por proporcionar el aceite de semilla vegetal Jatropha curcas para llevar a cabo el trabajo experimental. Los autores también agradecen al Instituto de Ingeniería Química y Tecnología, Universidad del Punjab, Lahore, Pakistán por el análisis GC-MS de aceites de semillas vegetales.

\section{Referencias}

Ahmed, M. y col. (2015). «Biernat, Biofuels - Status and Perspective». En: Intech. Cap. Pros- 
pects for the Production of Biodiesel in Pakistan, págs. 1-25.

Ali, M. (2016). Biological process- fuel. December 15, 2019, The News. URL: https:/ / bit.ly/3fL03uB.

Ali, M., B. Naqvi e I. Watson (2018). «Possibility of converting indigenous Salvadora persica L. seed oil into biodiesel in Pakistan». En: International Journal of Green Energy 15.7, 427-435. Online:https://bit.ly/3kF2uTc.

Ali, M. y A. Shaikh (2012). «Emission testing of jatropha and pongamia mixed biodiesel fuel in a diesel engine». En: NED University Journal of Research Thematic Issue on Energy, 43-52.Online:https://bit.ly/33WgamR.

Aransiola, E. y col. (2019). "Azad, Advances in Eco-Fuels for a Sustainable Environment». En: London: Woodhead Publishing. Cap. Prospective ecofuel feedstocks for sustainable production. Págs. 43-87.

Arunkumar, M. y col. (2018). «Performance study of a diesel engine with exhaust gas recirculation (EGR) system fuelled with palm biodiesel». En: International Journal of Thermal Engineering 6, 1-9. Online:https:/ / bit.ly /2DRVUrm.

Berchmans, H. y S. Hirata (2008). «Biodiesel production from crude Jatropha curcas L. seed oil with a high content of free fatty acids». En: Bioresource technology 99.6, 1716-1721. Online:https:/ / bit. ly/3gN9r1W.

Calder, J., M. Roy y W. Wang (2018). «Performance and emissions of a diesel engine fueled by biodiesel-diesel blends with recycled expanded polystyrene and fuel stabilizing additive». En: Energy 149, 204-212. Online:https : / / bit . ly / 3kGYe5m.

Chakrabarti, M. y M. Ali (2008). «Engine emissions testing of indigenous biodiesel/diesel fuel blends in Pakistan». En: NED Univ. J. Res 5.2, 1-9. Online:https:/ / bit.ly/3agMEci.

- (2009). «Performance of compression ignition engine with Indigenous castor oil bio diesel in Pakistan». En: NED university Journal of research 6.1, 10-20. Online:https://bit.ly/2Cowdyo.

Chauhan, S. y A. Shukla (2011). «Environmental Impacts of Production of Biodiesel and Its
Use in Transportation Sector». En: Croatia: InTech. Cap. Environmental Impact of Biofuels, págs. 1-13.

Climate Change Indicators (2015). Climate Change Indicators: Greenhouse Gases. Retrieved May 30, 2020. EPA. URL: https://bit.ly/33Q426H.

DAWN (2019). High Speed Diesel (HSD) sales to 6.764 $m$ tonnes. Retrieved May 30, 2020. Dawn Newspaper. URL: https:/ / bit.ly/3itfJEu.

Dincer, K. (2008). «Lower emissions from biodiesel combustion». En: Energy Sources, Part A 30.10, 963-968. Online:https:/ / bit.ly/3kE8RG1.

Hassan, M. H. y M. A. Kalam (2013). «An overview of biofuel as a renewable energy source: development and challenges». En: Procedia Engineering 56.39, 53. Online:https:// bit.ly/2POTIni.

Mall, A. P. (2015). «Performance and Emission Testing of Neem Oil Methyl Ester (Biodiesel)». En: nternal Combustion Engine. International Journal of Innovative Research in Science, Engineering and Technology 4.11, 11101-11111. Online:https:/ / bit. ly/31LRoD5.

Nair, Jayashri, Ajay K. Kaviti y Arun K. Daram (2017). "Analysis of performance and emission on compression ignition engine fuelled with blends of neem biodiesel». En: Egyptian Journal of Petroleum 26.4, 927-931. Online:https:/ / bit.ly / 3alfYyH.

Pervaz, M. A. (2018). Pakistan Energy Yearbook 2017. Inf. téc. Islamabad: Ministry of Energy (Petroleum Division), Hydrocarbon Development Institute of Pakistan.

Qamar, M. y col. (2020). «Techno-spatial assessment of waste cooking oil for biodiesel production in Pakistan». En: SN Applied Sciences 2, 1-16. Online:https://bit.ly/2FkmHOh.

Rao, T., G. Rao y K. Reddy (2008). «Experimental investigation of pongamia, jatropha and neem methyl esters as biodiesel on CI engine». En: Jordan Journal of Mechanical and Industrial Engineering 2.2, 117-122. Online:https: / / bit . ly / $3 \mathrm{gVkO} 87$.

Yuosafzai, F. (2018). 7000MW shortfall triggers extra unscheduled loadshedding. February 2019. URL: https://bit.ly/31QHtvW. 